\title{
17. Intraventricular Infusion Manometric Test for Measurement of CSF Absorption in Hydrocephal in Patients
}

\author{
Hiroshi Yamada, M.D. Masahiro Nagaya, M. D. \\ Masataka TAJIMA, M. D. \\ Department of Neurosurgery \\ Department of Surgery \\ Aichi Prefectural Colony, Central Hospital \\ Department of Neurosurgery \\ Nagoya Universaty School of Medicine
}

A large number of hydrocephalus may arrest spontaneously but there is little known about what consitutes arrested hydrocephalus. Recently the concept of normal pressure hydrocephalus developed and certain pediatric patients with normal cerebrospinal fluid pressure will have improvement after ventriculoatrial shunting procedure. Therefore it is important to neurosurgeons to know which cases the operative treatment is indicated. Furthermore the ventricle dilatation due to cerebral atrophy, hydrocephalus ex vacuo, is seen quite often in pediatric patients, and the differentiation is sometimes difficult.

Tests used for the evaluation of the cerebrospinal fluid dynamics include pneumoencephalography and isotope techniques, but these methods, however, only provide qualitative information. The purpose of this paper is to investigate the quantitative estimate of cerebrospinal fluid absorption of the hydrocephalic patients to know the indication of the operative treatment.

Technique: The right lateral ventricle is punctured through the fontanelle or burr hole with a $17 \mathrm{Ga}$. Teflon needle. The needle is connected with an electromanometer using an inductance transduser. The left lateral ventricle is punctuered with a $21 \mathrm{Ga}$. spinal needle. After recording the initial pressure, normal saline at $37^{\circ} \mathrm{C}$ is infused in lateral ventricle rapidly, within 30 to 60 seconds, until the pressure elevates to 750 to $1000 \mathrm{mmH}_{2} \mathrm{O}$. The monitor recording is continued until the pressure return to the original pressure. This test required about 30 minutes, and the patients were well tolerated with Ketalar anesthesia. In most of the present cases the high ventricular pressure continued only one or two minutes after the saline infusion and we have not observed any complications from this study.

Result: This test has been used in 18 cases of hydrocephalic patients between 4 months to 5 years who were thought that the cerebrospinal fluid pressure is not elevated or hydrocephalus is arrested clinically. In the cases of hydrocephalus ex vacuo, the pressure returned rapidly to normal level within one to two minutes. In arrested hydrocephalus it took about three minutes. But incompletely arrested cases it took over five minutes, and in some cases the pressure stayed above the original pressure even after ten minutes. In two cases the clinaical diagnosis was hydrocephalus ex vacuo. but the abosrption curve suggested pressure hydrocephalus. In ten cases 
among eighteen patients the ventriculoatrial shunting procedure was performed. Remarkable postoperative improvement was obtained in patients with elongated absorption time, over five minutes. But in two cases with short absorption time, within two minutes, the symptomatic improvement was not observed.

\title{
18. Follow Up Studies of Expanding Graniotomy for Infants and Children with Graniostenotic or Microcephalic State
}

\author{
Bun-ichiro Nagao, Yoshiaki Saito, Shozo Kawai \\ Ken-ichi Yamaga, Yutaka Hattori and Yutaka Hori \\ 2nd Department of Surgery, Nara Medical University
}

A seven years follow up study has been made on the expanding craniotomy for the infants and children with craniostentic and/or microcephalic state.

The new operative techniques, "expanding craniotomy" was brought foreward by Hori et al in 1965. In this method, several metalic springs are inserted into the groove made by linear craniotomy. The number of these springs is decided on the basis of E. E. G. findings.

As shown in the table 1 and 2, the two third of all cases show satisfactory improvements. The most important thing through these results is that improvements are seen even in cases with mental and motor maldevvelopment after the surgery. A five years follow up study of the expanding craniotomy revealed that this kind of surgery is effective for epileptic seizures $(33 \%)$, spastic paralysis $(21 \%)$ and stridor $(9 \%)$.

Stress is to be given to the improvements for the disturbances of autonomic nervous system, such as appetite, countenance, color and texture of skin, sweating, salivation, emotion and body supporting.

Comments:

1. Effects for the epileptic attack, nodding spasm and spasticity of extremities are to be shown one or two months after expanding craniotomy. The longer the time goes by after the surgery, the more the pleasant changes increase.

2. The expanding craniotomy is effective for the retardations of mental and motor developments, however, those changes are seen not in rush.

3. It is to be expected that this kind of surgery is fairly effective not only for "primary (congenital) and secondary (acquired) craniostenosis", but for "secondary microcephalus" and also "primary microcephalus in a slight grade".

4. As the result of normalization of autonomic nervous dysfunction, which follows after the surgery, those patients have better appetite and good humor, that is why we can expect those pitiful infants are going to be cheerfully healthy naughty kids. 